\title{
Humulus japonicus extract exhibits antioxidative and anti-aging effects via modulation of the AMPK-SIRT1 pathway
}

\author{
BOKYUNG SUNG ${ }^{1 *}$, JI WON CHUNG ${ }^{1 *}$, HA RAM BAE ${ }^{1}$, JAE SUE CHOI ${ }^{2}$, \\ CHEOL MIN KIM ${ }^{3}$ and NAM DEUK KIM ${ }^{1}$ \\ ${ }^{1}$ Department of Pharmacy, Molecular Inflammation Research Center for Aging Intervention, Pusan National University, \\ Busan 609-735; ${ }^{2}$ Faculty of Food Science and Biotechnology, Pukyong National University, Busan 608-737; \\ ${ }^{3}$ Department of Biochemistry, School of Medicine, Pusan National University, \\ Yangsan, Gyeongsangnam-do 626-770, Republic of Korea
}

Received November 4, 2014; Accepted January 28, 2015

DOI: $10.3892 /$ etm.2015.2302

\begin{abstract}
The perennial herb, Humulus japonicus, has been previously described as possessing potential antituberculosis and anti-inflammatory properties. In the present study, the anti-aging activity of ethanol extracts from the leaves of $H$. japonicus (HJE) was evaluated in yeast and human fibroblast cells. In addition, the antioxidant activity of HJE was analyzed using free radical scavenging assays. Furthermore, the mechanism underlying the hypothesized HJE-associated extension of lifespan was investigated, and the results indicated that HJE was able to extend the lifespan of yeast cells. Further experiments demonstrated that HJE upregulated the longevity-associated proteins, sirtuin 1 and AMP-activated protein kinase, and effectively inhibited the generation of reactive oxygen species (ROS). In addition, the antioxidative potential of the active constituents of HJE, including luteolin, luteolin 7-glycoside, quercetin and quercitrin, was evaluated and the results demonstrated that these flavonoids were able to scavenge ROS in cell-free and intracellular systems. In summary, the results revealed that HJE possessed the potential for antioxidative activity; however, further in vivo investigations are required with the aim of developing safe, high-efficacy anti-aging agents.
\end{abstract}

Correspondence to: Professor Nam Deuk Kim, Department of Pharmacy, Molecular Inflammation Research Center for Aging Intervention, Pusan National University, 2 Busandaehak-ro 63 Beon-gil, Busan 609-735, Republic of Korea

E-mail: nadkim@pusan.ac.kr

${ }^{*}$ Contributed equally

Key words: Humulus japonicus, reactive oxygen species, flavonoid, lifespan, aging

\section{Introduction}

Aging is associated with the deterioration of a number of physiological processes, leading to a decline in functional capabilities, which ultimately impacts the health and overall function of an organism. Among humans and other mammals, these deteriorations occur primarily in the immune system, and result in an increased susceptibility to various conditions, including chronic inflammation, muscle loss, cancer and age-associated degenerative disorders (1). Extensive research over the five previous decades has focused on identifying the underlying mechanisms of aging. In addition to understanding the mechanisms of the aging process, one of the principal aims of research into aging is the identification of intervention strategies or the development of therapeutics that enhance longevity.

It is widely acknowledged that the limitation of calorie intake, also known as calorie restriction (CR), may increase the mean lifespan of an organism by up to $60 \%$, while reducing the incidence of degenerative disease (2). Although the positive effects of $\mathrm{CR}$ on slowing the aging process and increasing the lifespan have been demonstrated in a range of species, CR has not been widely adopted as a preventative strategy due to the difficulty of following such a strict dietary regime (3). Therefore, there is a requirement for the identification of small molecules that mimic the effect of CR without the application of a strict diet, or that modulate the molecular pathways responsible for the anti-aging effect produced by $\mathrm{CR}$, in order to slow aging and increase the lifespan of an organism (3). The molecular targets, sirtuin 1 (SIRT1) and AMP-activated protein kinase (AMPK), are potential candidates. SIRT1 is a mammalian ortholog of the yeast protein, silent information regulator 2, and increased activity levels of SIRT1 by activators, such as resveratrol, have been observed to extend the lifespan in a number of species (4-6). Similarly, AMPK has been recognized as a potential molecular target for the regulation of longevity (7). The progression of the aging process has been shown to correlate with a reduction in the activity of SIRT1 and AMPK. Thus, pharmacological interventions aimed at regulating SIRT1 and AMPK may provide effective methods for improving health in aging patients and extending their lifespan $(4,5,7)$. 
Medicinal plants have been used for millennia in numerous cultures to prevent and treat of a variety of diseases. However, the active constituents of these medicinal plants and their precise mechanisms of action are not fully understood. Humulus japonicus Siebold et Zucc, from the Cannabaceae family, is an example of such plants. H. japonicus is a perennial herb that grows commonly as a weed in Korea and China, where it is also known as 'Japanese hop'. In Western countries, H. japonicus was previously imported for ornamental purposes; however, the plant is considered to be an invasive plant in numerous countries due to its notable survival capacity. In traditional Chinese medicine, $H$. japonicus has been used to treat pneumonia, diarrhea, hypertension, leprosy and tuberculosis. In Korea, the leaves of $H$. japonicus have been used in the treatment of pulmonary tuberculosis, tuberculosis cervical lymphadenitis and hypertension $(8,9)$. In addition, previous studies have indicated that the extract of $H$. japonicus (HJE) possesses antioxidative, antibacterial, antimycobacterial, antimutagenic, anti-inflammatory and antitumor properties (9-14). Over the previous five decades, a number of the bioactive constituents from $H$. japonicus have been identified and reported, including terpenes, lupulones, phenolics and flavonoids $(8,11,15,16)$.

To the best of our knowledge, the potential of HJE to extend the lifespan and its effect on the aging process have not yet been investigated. Thus, the aim of the present study was to investigate the effect of HJE on lifespan, and to elucidate the signaling pathways and active constituents involved in lifespan extension. In addition, the antioxidant capacities of HJE and its active constituents were evaluated, since reactive oxygen species (ROS) are a major contributing factor to the aging process.

\section{Materials and methods}

Chemicals and reagents. Luteolin, luteolin 7-glucoside, quercetin, quercitrin and resveratrol were obtained from Sigma-Aldrich (St. Louis, MO, USA), dissolved in ethanol and stored at $-20^{\circ} \mathrm{C}$ until required. In addition, 6-hydroxy-2,5,7,8-tetramethylchroman-2-carboxylic acid (Trolox), 3-morpholinosydnonimine hydrochloride (SIN-1) and carboxy- $\mathrm{H}_{2}$ DCFDA were obtained from Sigma-Aldrich. Dulbecco's modified Eagle's medium (DMEM), fetal bovine serum (FBS) and penicillin-streptomycin were purchased from GE Healthcare (HyClone; Logan, UT, USA). Rabbit polyclonal antibodies against phospho-AMPK $\alpha 1 / 2$ (Thr 172; cat. no. sc-33524), SIRT1 (cat. no. sc-15404) transcription factor IIB (cat. no. sc-225) and mouse monoclonal $\beta$-actin (cat. no. sc-47778), and goat anti-rabbit IgG-horseradish peroxidase (HRP)-conjugated (cat. no. sc-2004) and anti-mouse IgG-HRP-conjugated (cat. no. sc-2031) antibodies were obtained from Santa Cruz Biotechnology, Inc. (Santa Cruz, CA, USA). 2',7'-Dichlorodihydrofluorescein diacetate $\left(\mathrm{H}_{2}\right.$ DCFDA) was purchased from Invitrogen Life Technologies (Eugene, OR, USA).

Plant materials and extraction. Fresh leaves of Acanthopanax sessiliflorus, Rubus crataegifolius (Bunge), Vitis thunbergii var. sinuata and $H$. japonicus were collected in Busan (Korea) and authenticated by Professor JS Choi at Pukyong National University (Busan, Korea). These plant specimens were deposited in Professor Hae Chung's labora- tory (Pusan National University, Busan, Korea). The fresh leaves of these plants were dried, chopped into small pieces and refluxed with absolute ethanol (EtOH). The extract of each plant was separated from the residues through Whatman No. 1 filter paper (GE Heathcare Life Sciences, Pittsburgh, PA, USA), then concentrated to dryness to render the EtOH extract. The extract was subsequently suspended in EtOH and stored at $-20^{\circ} \mathrm{C}$ until required.

Yeast strain and microbiological methods. A BY4742 yeast strain (MAT $\alpha$ his $3 \Delta 1$ leu2 $\Delta 0$ lys $2 \Delta 0$ ura $3 \Delta 0$; EUROSCARF, Frankfurt, Germany) was used for chronological lifespan (CLS) measurements, as described previously (17). Yeast was grown to exponential phase in rich yeast, peptone, dextrose medium or in synthetic defined minimal medium (Sigma-Aldrich), both containing $2 \%$ glucose, which were prepared as described by Sherman (18). CLS measurements were performed as previously described (19).

Inhibition of total ROS generation. The scavenging activity of the agents under investigation was assessed using $\mathrm{H}_{2}$ DCFDA, a fluorescent oxidative stress indicator. For the measurement of ROS-scavenging activity in a cell-free system, $\mathrm{H}_{2}$ DCFDA was mixed with esterase ( $\mathrm{pH} 7.4)$ and incubated for $20 \mathrm{~min}$ at $37^{\circ} \mathrm{C}$. The mixture was then placed on ice in the dark until immediately prior to measurement. $\mathrm{H}_{2} \mathrm{DCFDA}$ was hydrolyzed to non-fluorescent 2',7'-dichlorodihydrofluorescein (DCFH) by esterase (Sigma-Aldrich) and subsequently oxidized to highly fluorescent 2',7'-dichlorofluorescein by the $\mathrm{ROS}, \cdot \mathrm{O}_{2}{ }^{-}(20)$. The fluorescence intensity of the oxidized DCFH was quantified using a GENios fluorescence microplate reader (Tecan Group Ltd., Männedorf, Switzerland) at excitation and emission wavelengths of 485 and $530 \mathrm{~nm}$, respectively. Measurement was performed for $30 \mathrm{~min}$, with or without the addition of SIN-1 as an $\mathrm{O}_{2}{ }^{-}$donor. In addition, a similar experiment was performed using Trolox as a positive control to compare for antioxidant capacity.

Cell culture. Human fibroblast Hs27 (CRL-1634) cells were obtained from the American Type Culture Collection (Manassas, VA, USA). The cells were cultured in DMEM containing $10 \% \mathrm{FBS}$, penicillin $(100 \mathrm{U} / \mathrm{ml})$ and streptomycin $(100 \mu \mathrm{g} / \mathrm{ml})$ at $37^{\circ} \mathrm{C}$ in a humidified atmosphere of $5 \% \mathrm{CO}_{2}$ in air. The fibroblast cells were plated at $90-95 \%$ confluency for all the experiments.

Inhibitory activityon intracellular ROS generation. Intracellular ROS generation was measured using carboxy- $\mathrm{H}_{2}$ DCFDA, a cell-permeable dye. This compound is oxidized intracellularly by ROS to form fluorescent DCF. Briefly, the Hs27 cells were incubated for $24 \mathrm{~h}$ in a 96 -well plate. After one day, the medium was replaced with fresh serum-free medium containing HJE or flavinoids. The cells were pretreated with HJE or flavonoids for $1 \mathrm{~h}$ and were then exposed to ultraviolet B (UVB), according to designated experimental conditions. UVB irradiation was carried out using a UV Crosslinker (CL-1000; UVP, LLC, Upland, CA, USA) at the desired intensity $\left(100 \mathrm{~J} / \mathrm{m}^{2}\right)$. Prior to UVB exposure, the cells were washed with phosphate-buffered saline (PBS) and resusupended in fresh PBS. Subsequently, the cells were incubated with $10 \mu \mathrm{M}$ carboxy- $\mathrm{H}_{2}$ DCFDA for $10 \mathrm{~min}$ 

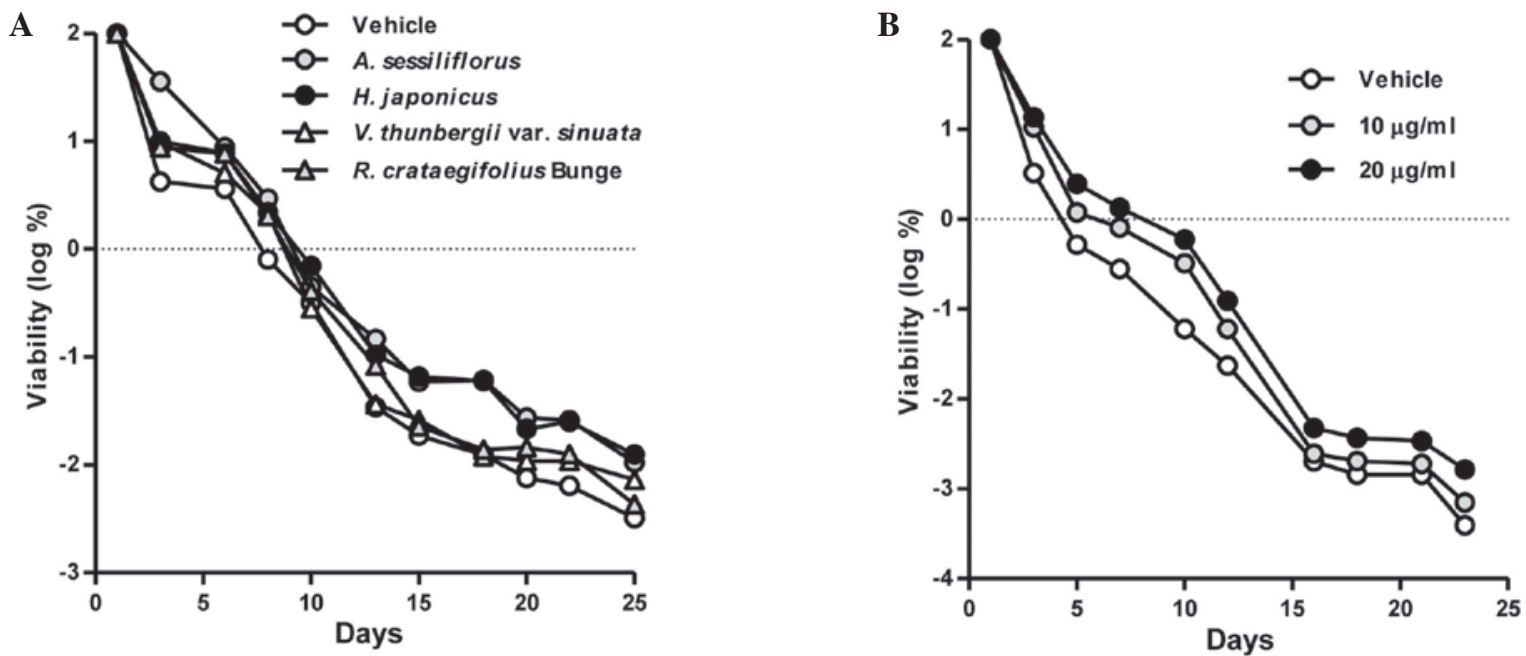

Figure 1. Effect of Humulus japonicus extract (HJE) on yeast lifespan. Extracts were added to media, and yeast viability (corresponding to chronological life span) was determined by counting the number of colony-forming units (CFUs). (A) Effects of the ethanolic extracts (10 $\mu \mathrm{g} / \mathrm{ml})$ of Acanthopanax sessiliflorus, HJE, Vitis thunbergii var. sinuata and Rubus crataegifolius (Bunge) on Saccharomyces cerevisiae lifespan. (B) Yeast cells were cultivated with the various concentrations of HJE and the effect on lifespan was assessed by counting the number of CFUs.

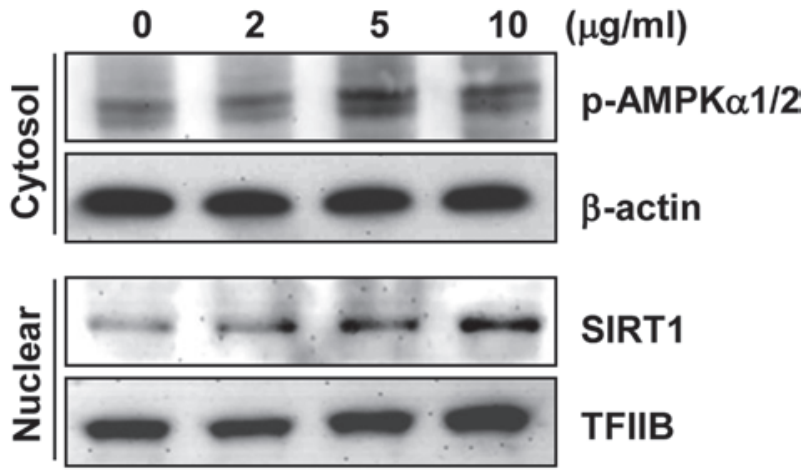

Figure 2. Effects of Humulus japonicus extract (HJE) on the activation of AMPK and SIRT1 in Hs27 cells. Cells were treated with the indicated concentration of HJE for $24 \mathrm{~h}$. Cytosolic protein levels of AMPK and nuclear protein levels of SIRT1 were determined by western blot analysis. $\beta$-actin and TFIIB were used as loading controls for the cytosolic and nuclear proteins, respectively. TFIIB, transcription factor IIB; p-AMPK, phospho-AMP-activated protein kinase; SIRT1, sirtuin 1.

at $37^{\circ} \mathrm{C}$, and washed twice with PBS. Modulations in fluorescence intensity were measured every 5 min for $30 \mathrm{~min}$ using a GENios fluorescence plate reader, at excitation and emission wavelengths of 485 and $530 \mathrm{~nm}$, respectively.

Cytosolic and nuclear extract preparations. Cells were washed with ice-cold PBS and harvested. A buffer containing $10 \mathrm{mM}$ Tris (pH 8.0), $1.5 \mathrm{mM} \mathrm{MgCl}_{2}, 1$ mM DTT, $0.1 \%$ Nonidet P-40 and protease inhibitors was used to extract the cytosolic fractions by centrifugation at $14,000 \mathrm{x}$ g for $15 \mathrm{~min}$ at $4^{\circ} \mathrm{C}$. Nuclear fractions were extracted from the resulting pellets using a buffer containing $10 \mathrm{mM}$ Tris (pH 8.0), $50 \mathrm{mM} \mathrm{KCl,} 100 \mathrm{mM} \mathrm{NaCl}$ and protease inhibitors. Aliquots of the cytosolic or nuclear extracts were boiled in gel loading buffer (Bio-Rad Laboratories, Inc., Hercules, CA, USA) for $5 \mathrm{~min}$.

Western blot analysis. In order to determine the expression levels of the proteins under investigation, cell extracts were prepared and western blot analysis was conducted. In brief, cell extracts containing equal quantities of proteins $(20 \mu \mathrm{g})$ were subjected to $8-10 \%$ sodium dodecyl sulfate polyacrylamide gel electrophoresis and transferred to polyvinylidene fluoride membranes (EMD Millipore, Billerica, MA, USA). The membranes were probed with the primary antibodies (1:1,000 dilutions) overnight at $4{ }^{\circ} \mathrm{C}$, followed by the HRP-conjugated secondary antibodies (1:5,000 dilutions) for $1 \mathrm{~h}$ at room temperature. Signals were detected using an enhanced chemiluminescence reagent (AbFrontier Co., Ltd., Seoul, Korea).

Statistical analysis. Analysis of variance was used to analyze the differences between each group, and Dunnett's multiple comparison test was used to determine the differences between the mean values of the groups. All statistical analyses were conducted using GraphPad Prism version 5.02 (GraphPad Software, Inc., San Diego, CA, USA). P<0.05 was considered to indicate a statistically significant difference.

\section{Results}

HJE extends the yeast lifespan. Effects of HJE on the lifespan of yeast were investigated. In traditional Korean medicine, plants with palmate (hand-shaped) leaves are considered to possess health benefits. Thus, extracts of A. sessiliflorus, a well-studied medicinal plant, $V$. thunbergii var. sinuata and $R$. crataegifolius (Bunge) were also examined. To evaluate the anti-aging effect of the plant extracts, yeast cells were cultivated with the extracts for 25 days and the CLS was measured by monitoring the number of colony-forming units (CFUs). The number of viable yeast cells that were able to reproduce and form colonies reduced in culture over time, regardless of the presence of plant extracts. As presented in Fig. 1A, the number of CFUs was higher in the HJE-treated cultures when compared with untreated control cultures between days 10 and 25 . However, cultivation with the extracts from A. sessiliflorus, V. thunbergii var. sinuata or 
A

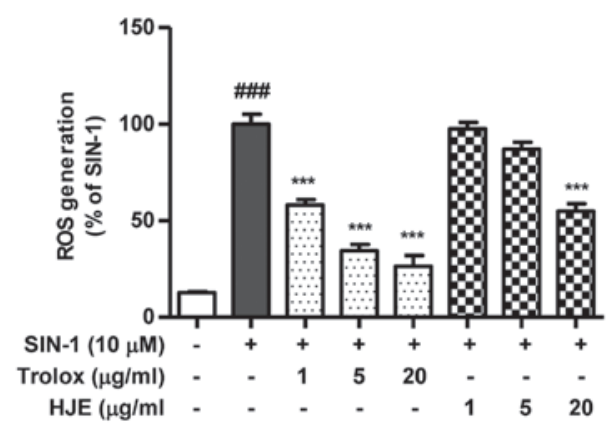

B

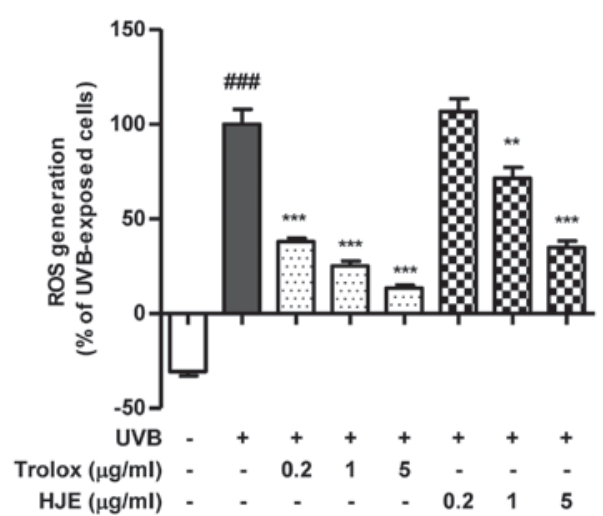

Figure 3. Effects of HJE on ROS generation. The inhibitory effect of HJE on ROS production was evaluated using a 2',7'-dichlorodihydrofluorescein diacetate assay to detect the ROS. Trolox, a well-known scavenger of ROS, was used as positive control. (A) Scavenging activity of HJE on ROS generation induced by $10 \mu \mathrm{M}$ SIN-1 was measured in vitro. ${ }^{\# \# \#} \mathrm{P}<0.001$, vs. untreated control; ${ }^{* *} \mathrm{P}<0.01$ and ${ }^{* * *} \mathrm{P}<0.001$, vs. $10 \mu \mathrm{M}$ SIN-1-treated control. (B) Hs27 cells were pretreated with HJE extract for $1 \mathrm{~h}$ and further treated with $10 \mathrm{~J} / \mathrm{m}^{2} \mathrm{UVB}$. ${ }^{\# \#} \mathrm{P}<0.001$, vs. untreated control; ${ }^{* * * *} \mathrm{P}<0.001$ vs. UVB-treated control. Results were analyzed by one-factor analysis of variance and are expressed as the mean \pm standard error. ROS, reactive oxygen species; HJE, Humulus japonicus extract; SIN-1, 3-morpholinosydnonimine hydrochloride; Trolox, 6-hydroxy-2,5,7,8-tetramethylchroman-2-carboxylic acid; UVB, ultraviolet B.

R. crataegifolius (Bunge) was shown to have no effect on the yeast lifespan.

Therefore, the HJE exhibited comparatively notable lifespan extension, and the effects of different concentrations of HJE on the yeast lifespan were subsequently examined. The results indicated that HJE increased the viability of yeast cells in a concentration-dependent manner (Fig. 1B). Collectively, these results indicated that HJE exerts a beneficial anti-aging effect.

HJE modulates the expression levels of AMPK and SIRT1. A number of previous studies have demonstrated that AMPK and SIRT1 serve important functions in the aging process (21-23). As HJE was observed to exert a beneficial effect on the lifespan of yeast, the effect of HJE on AMPK and SIRT1 expression was subsequently examined. AMPK is the principal energy sensor in eukaryotic cells and functions to maintain cellular energy homeostasis and mitochondrial biogenesis (24). Aging is associated with a reduction in AMPK-induced mitochondrial biogenesis, and the activation of AMPK has been observed to increase the lifespan of fruit flies $(25,26)$. The results of the present study indicated that HJE readily activated AMPK (Fig. 2).

SIRT1 is predominantly located in the nuclei and is responsible for oxidative stress. In addition, a decrease in nuclear SIRT1 levels has been previously reported in the hearts of aged mice $(27,28)$. Therefore, in the present study, nuclear proteins were employed for the detection of SIRT1 The results demonstrated that HJE treatment increased nuclear SIRT1 levels in a concentration-dependent manner (Fig. 2), indicating that HJE may extend the lifespan by modulating the expression levels of AMPK and SIRT1. However, further mechanistic experiments are required at the molecular and organism levels.

HJE inhibits ROS generation. The effect of HJE on ROS generation was evaluated to elucidate the mechanism underlying the HJE-mediated extension of yeast lifespan.
An equivalent concentration of Trolox, a water-soluble vitamin $\mathrm{E}$ analog, was used as a positive control for comparison with the inhibitory effect of HJE on SIN-1-induced ROS in a cell-free system. As presented in Fig. 3A, HJE appeared to scavenge the ROS generated by SIN-1 in a concentration-dependent manner. However, the HJE-induced inhibition of ROS generation was lower compared with that of Trolox.

As the antioxidative capacity of the testing molecules may have differed between cell-free and intracellular systems, the antioxidative effect of HJE was examined in Hs27 human fibroblast cells. UVB radiation has been reported to induce ROS generation, resulting in cellular senescence, and the role of SIRT1 in UVB-induced skin aging is well-established $(29,30)$. Thus, UVB was used to induce ROS generation in the Hs27 skin fibroblast cells. Pretreatment with HJE $(5 \mu \mathrm{g} / \mathrm{ml})$ resulted in a significant reduction (34.8\%) in the generation of ROS when compared with the UVB-exposed control cells (Fig. 3B). These results indicated that HJE effectively scavenged ROS in the cell-free system and UVB-stimulated fibroblast cells.

HJE-derived flavonoids suppress ROS generation. H. japon$i c u s$ is known to contain a number of flavonoids and phenolics that are responsible for various biological activities $(8,11,12)$. In order to investigate whether the antioxidative capacity of HJE is mediated by the aforementioned active constituents, the effects of luteolin, luteolin 7-glycoside, quercetin and quercitrin (structures shown in Fig. 4A) on oxidative stress were determined. The ROS scavenging activities of the HJE-derived flavonoids, at concentrations of $0.2,1$ and $5 \mu \mathrm{M}$, on the cell-free system are presented in Fig. 4B. Among the four tested compounds from HJE, luteolin 7-glucoside exhibited the highest ROS scavenging capacity.

Next, the intracellular antioxidative effect of HJE-derived flavonoids was investigated. UVB radiation was used to induce ROS generation in order to assess the capacities of the HJE-derived flavonoids to inhibit intracellular ROS. 
A

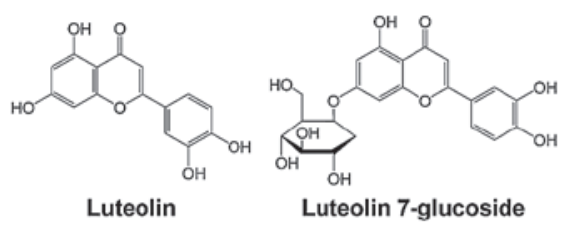<smiles>OC1CC(O)C2C(C1)OC(C1CCC(O)C(O)C1)C2O</smiles>

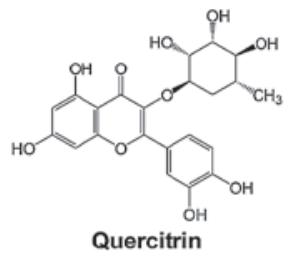

B
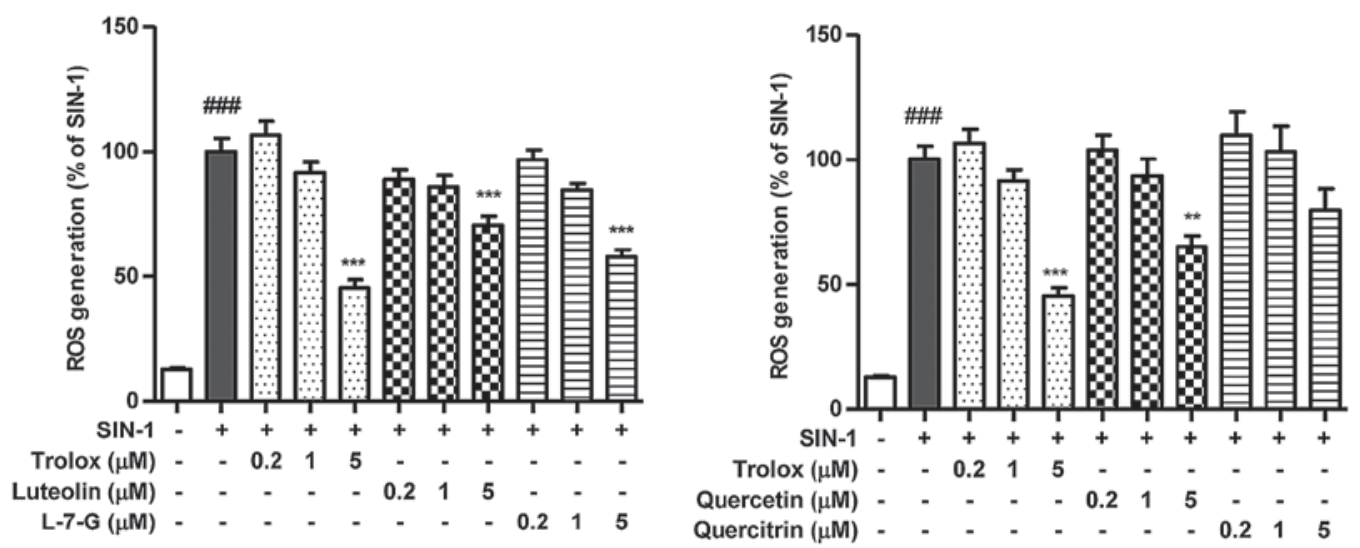

Figure 4. In vitro ROS scavenging activity of the active constituents of Humulus japonicus. The inhibitory effects of the active constituents from $H$.japonicus on ROS production were evaluated using a $2^{\prime}, 7^{\prime}$-dichlorodihydrofluorescein diacetate assay. (A) Chemical structures of the active constituents of $H$. japonicus. (B) Scavenging activity of the H. japonicus-derived flavonoids on ROS generation induced by $10 \mu \mathrm{M}$ SIN-1 was measured in vitro. ${ }^{\# \# \#} \mathrm{P}<0.001$, vs. untreated control; ${ }^{* *} \mathrm{P}<0.01$ and ${ }^{* * * *} \mathrm{P}<0.001$, vs. $10 \mu \mathrm{M}$ SIN-1-treated control. Results were analyzed by one-factor analysis of variance and are expressed as the mean \pm standard error. ROS, reactive oxygen species; L-7-G, luteolin 7-glucoside; Trolox, 6-hydroxy-2,5,7,8-tetramethylchroman-2-carboxylic acid; SIN-1, 3-morpholinosydnonimine hydrochloride.
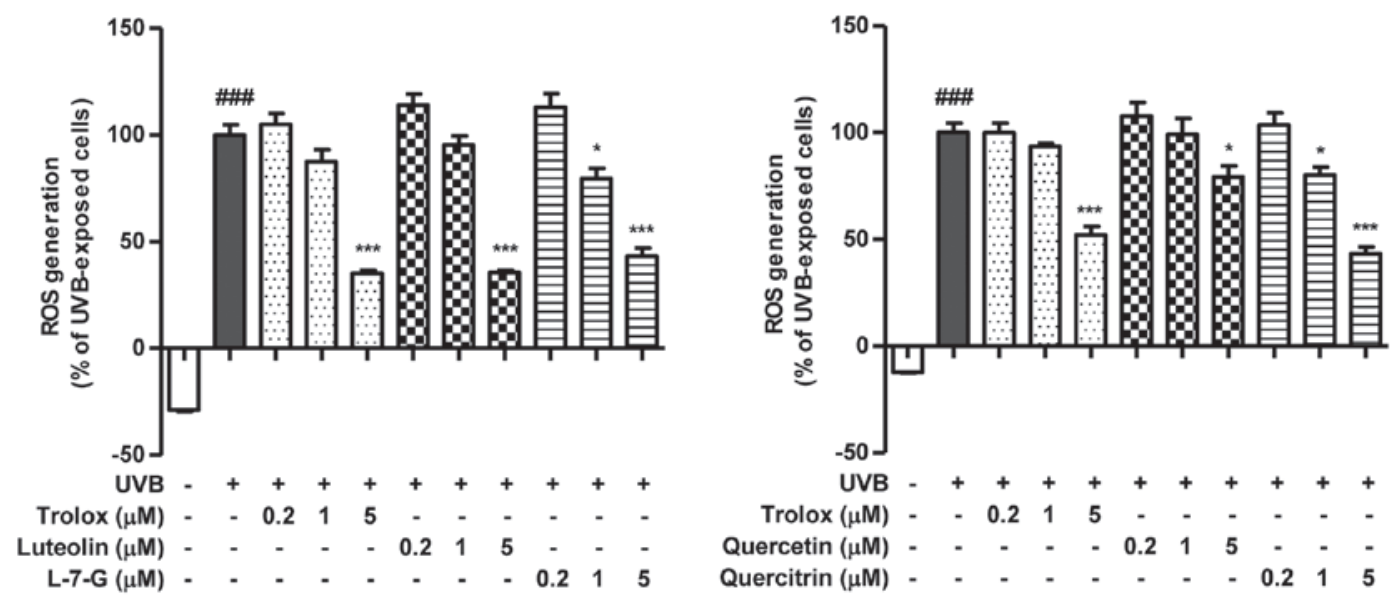

Figure 5. Effects of active constituents from Humulus japonicus on UVB-induced ROS generation in Hs27 cells. Cells were treated with the indicated concentration of Trolox, luteolin, luteolin 7-glucoside, quercetin or quercitrin for $1 \mathrm{~h}$, followed by treatment with $10 \mathrm{~J} / \mathrm{m}^{2} \mathrm{UVB}$. ROS generation was assessed by measuring the fluorescence intensity of $2^{\prime}, 7^{\prime}$-dichlorodihydrofluorescein diacetate following UVB exposure. Results are expressed as the mean \pm standard error. ${ }^{\# \# \# ~} \mathrm{P}<0.001$, vs. untreated control; " $\mathrm{P}<0.05$ and ${ }^{* * *} \mathrm{P}<0.001$, vs. UVB-treated control. L-7-G, luteolin 7-glucoside; ROS, reactive oxygen species; UVB, ultraviolet B; Trolox, 6-hydroxy-2,5,7,8-tetramethylchroman-2-carboxylic acid.

The scavenging activities of the active constituents on UVB-induced ROS in pretreated Hs27 cells are shown in Fig. 5. In particular, luteolin was observed to exert a marked ROS scavenging effect on intracellular ROS, while luteolin 7-glucoside was most effective at scavenging ROS in the cell-free system. Thus, the results indicated clear differ- ences in the antioxidative capacity among the HJE-derived flavonoids. The rank order was as follows: Luteolin > luteolin 7-glucoside $=$ quercetin $>$ quercitrin in the intracellular system. These results clearly demonstrated that the potent antioxidative properties of HJE may be mediated by flavonoids. 


\section{Discussion}

The present study aimed to investigate the effects of HJE on yeast lifespan. Furthermore, the effect of HJE on the expression levels of SIRT1 and AMPK, which are involved in lifespan modulation, was determined. In addition, as oxidative stress is a major contributing factor in the aging process, the antioxidative capacities of HJE and its active constituents were assessed.

To the best of our knowledge, the present study is the first to propose that HJE may be able to increase the life span and delay the detrimental health effects associated with aging. Previous studies have hypothesized that medicinal plants, such as Lithospermum erythrorhizon, Panax ginseng, Ginkgo biloba and Rhodiola rosea, may exert beneficial effects on cellular senescence and longevity, which are associated with the aging process (31-34). Although $H$. japonicus has been described and used as a traditional remedy in Korea and China, limited information is available with regard to the underlying biological activities, active phytochemicals and action mechanisms of this plant.

In the present study, HJE was observed to activate AMPK in human fibroblast cells. AMPK has been demonstrated to serve a key function in the process of aging and the determination of lifespan (35). Overexpression of AMPK has been associated with prolonged lifespans in Caenorhabditis elegans and the Drosophila fruit fly $(26,36)$. In addition, Greer et al demonstrated that the presence of AMPK is essential for lifespan extension by $\mathrm{CR}$ in C. elegans via phosphorylation of the FOXO transcription factor (37). Notably, AMPK has been reported to phosphorylate FOXO3 in mammalian cells, indicating that the modulation of FOXO by AMPK may be conserved among species (38). Thus, these studies indicate that the activation of AMPK is involved in the extension of lifespan (36,35-38). The results of the present study are consistent with those of previous studies, which have demonstrated that small molecules, such as chicoric acid and metformin, are able to prolong lifespan in worms via modulation of AMPK expression $(39,40)$. Furthermore, previous studies have reported that quercitrin, quercetin and luteolin, the active constituents of HJE, activate AMPK, which indicates that these active constituents may contribute to the effect of HJE on AMPK expression levels (41-43).

An additional possible mechanism for the life-extending effect of HJE involves SIRT1. The function of sirtuins in lifespan modulation in yeast was recognized over a decade ago; however, the capacity of sirtuins to extend lifespans in other organisms remains controversial. There are seven sirtuin homologs (SIRT1-7) in mammals, of which SIRT1 is the most extensively studied. In mammals, the anti-aging mechanism underlying CR has been shown to involve the activation of SIRT1 in numerous tissues (44). Thus, increased expression of SIRT1 in mice results in phenotypes that resemble the lifespan-extending effects of CR (45). Since SIRT1 performs a key function in lifespan modulation, the protein has attracted increasing attention as a potential drug target for delaying the onset of aging and extending the lifespan. For example, the polyphenol, resveratrol, which has been identified in red wine and grapes, targets SIRT1 and exerts a beneficial effect on lifespan $(5,6)$. Furthermore, reduced levels of SIRT1 have been observed in aged mouse heart tissue (28). Therefore, it is possible that the lifespan-extending effect of HJE is mediated by SIRT1 regulation. However, the effect exerted by HJE on the lifespans of higher order organisms is yet to be fully elucidated.

A recognized mechanism underlying the aging process is the accumulation of oxidative damage; a hypothesis that has been widely accepted (46). Thus, an antioxidative effect may result in lifespan extension. For example, the ability of resveratrol to function as an antioxidant is a possible alternative mechanism for its lifespan-extending effect, other than the activation of sirtuins (47). Results from the present study and previous studies indicate that HJE and its active flavonoid constituents exhibit antioxidative activity $(10,12,13)$. Notably, a discrepancy in the antioxidative activity of these active constituents was observed between cell-free and cell culture systems. The aglycone forms of the flavonoids (luteolin and quercetin) exerted more potent ROS scavenging activities in Hs27 cells when compared with their sugar-conjugated forms (luteolin 7-glucoside and quercitrin). The results a previous study demonstrated that the antioxidant activity of flavonoids is determined by the position, number and state of the hydroxyl groups located on the benzene ring, and that the glycosylation of these hydroxyl groups results in a reduction in antioxidative capacity (48). Thus, the role of the sugar moiety in the antioxidative activity of flavonoids remains controversial, and depends on the type and location of the sugar group. Although luteolin exerted the strongest ROS scavenging capacity, quercetin is the most extensively studied molecule among the active constituents of $H$. japonicus. Cheng et al reported that the antioxidative activity of quercetin in C. elegans was enhanced if the molecule was sugar-conjugated (49). Furthermore, the authors proposed that the antioxidant effect may be attributable to the moiety promoted by the rhamnopyranoside, which is able to facilitate flavonoid absorption, as described in the animal model $(49,50)$. However, Comalada et al reported that the anti-inflammatory effect exerted by quercitrin appeared to be mediated by the release of quercetin, which was generated by glycoside cleavage in rat intestinal microbiota (51). In accordance with these observations, Jiang et al demonstrated that quercetin is a major metabolite of quercitrin, and that the production of quercetin was able to improve the absorption rate and bioavailability of quercitrin in vivo (52). These results may explain the finding that aglycone forms of HJE-derived flavonoids exhibit stronger antioxidative capacities compared with those of the sugar-conjugated forms in a cell culture system.

In conclusion, the results obtained in the present study demonstrated that pretreatment with HJE enhanced the lifespan of yeast. Furthermore, HJE was shown to exert antioxidant activities in a cell-free system and in human fibroblast cells. In addition, the active constituents of $H$. japonicus, namely luteolin, luteolin 7-glucoside, quercetin and quercitrin, exhibited antioxidative capacities, with luteolin exerting the most notable ROS scavenging activity among the tested constituents. Thus, the results of the present study indicate that HJE may have the potential to be used as a source for the development of pharmacological or nutraceutical interventions that delay the aging process and extend longevity. However, further studies in animals and humans are required to fully determine the potential of this medicinal plant for improving human health. 


\section{Acknowledgements}

The study was supported by grants from the Research and Development Program of the Ministry of Trade, Industry and Energy (MOTIE)/Korea Institute for Advancement of Technology (no. N0000697; Establishment of Infrastructure for Anti Aging Industry Support) and the Research and Development Program of MOTIE/Korea Evaluation Institute of Technology (no. 10040391; Development of Functional Food Materials and Device for Prevention of Aging Associated Muscle Function Decrease). The authors thank the Aging Tissue Bank (Busan, Korea) for providing research information.

\section{References}

1. López-Otín C, Blasco MA, Partridge L, Serrano M and Kroemer G: The hallmarks of aging. Cell 153: 1194-1217, 2013.

2. Weindruch R, Walford RL, Fligiel S and Guthrie D: The retardation of aging in mice by dietary restriction: longevity, cancer, immunity and lifetime energy intake. J Nutr 116: 641-654, 1986.

3. Mair W and Dillin A: Aging and survival: the genetics of life span extension by dietary restriction. Annu Rev Biochem 77 727-754, 2008

4. Howitz KT, Bitterman KJ, Cohen HY, et al: Small molecule activators of sirtuins extend Saccharomyces cerevisiae lifespan. Nature 425: 191-196, 2003.

5. Valenzano DR, Terzibasi E, Genade T, Cattaneo A, Domenici L and Cellerino A: Resveratrol prolongs lifespan and retards the onset of age-related markers in a short-lived vertebrate. Curr Biol 16: 296-300, 2006.

6. Baur JA, Pearson KJ, Price NL, et al: Resveratrol improves health and survival of mice on a high-calorie diet. Nature 444 337-342, 2006

7. McCarty MF: Chronic activation of AMP-activated kinase as a strategy for slowing aging. Med Hypotheses 63: 334-339, 2004.

8. Yu BC, Yang MC, Lee KH, Kim KH, Choi SU and Lee KR Two new phenolic constituents of Humulus japonicus and their cytotoxicity test in vitro. Arch Pharm Res 30: 1471-1475, 2007.

9. Hong M, Son E, Lee S, et al: Anti-mycobacterial effects of the extract of Humulus japonicus. Han'guk Sikp'um Kwahakhoe chi 46: 94-99, 2014 (In Korean).

10. Park SW, Woo CJ, Chung SK and Chung KT: Antimicrobial and antioxidative activities of solvent fraction from Humulus japonicus. Han'guk Sikp'um Kwahakhoe chi 26: 464-470 1994 (In Korean).

11. Park SW, Kim SH and Chung SK: Antimutagenic effects and isolation of flavonoids from Humulus japonicus extract. Han'guk Sikp'um Kwahakhoe chi 27: 897-901, 1995 (In Korean).

12. Park SW, Chung SK and Park JC: Active oxygen scavenging activity of luteolin-7-O-b-D-glucoside isolated from Humulus japonicus. Han'guk Sikp'um Yŏngyang Kwahakhoe chi 29: 106-110, 2000 (In Korean).

13. Lee YR, Kim K, Lee SH, Kim MY, Park HJ and Jeong HS: Antioxidant and antitumor activities of methanolic extracts from Humulus japonicus. Han'guk Sikp'um Yŏngyang Hakhoe chi 25 357-361, 2012 (In Korean).

14. Hwang S,JungH,Jang W,JoM,Kim SandJeeS: Anti-inflammatory effects of the $\mathrm{MeOH}$ extract of Humulus japonicus in vitro. Han'bang An IIbi Inhu P'ibu Kwahakhoe chi 22: 71-91, 2009 (In Korean).

15. Aritomi M: Studies on the chemical constituents in leaves of Humulus japonicus Siebold et Zuccarini. Yakugaku Zasshi 82: 1331-1332, 1962 (In Japanese).

16. Naya Y and Kotake M: The constituents of Hops. V. The volatile composition of Humulus japonicus Sieb. et Zucc. Bull Chem Soc Jpn 43: 3594-3596, 1970.

17. Brachmann CB, Davies A, Cost GJ, et al: Designer deletion strains derived from Saccharomyces cerevisiae S288C: a useful set of strains and plasmids for PCR-mediated gene disruption and other applications. Yeast 14: 115-132, 1998.

18. Sherman F: Getting started with yeast. Methods Enzymol 350: $3-41,2002$.

19. Alvers AL, Fishwick LK, Wood MS, et al: Autophagy and amino acid homeostasis are required for chronological longevity in Saccharomyces cerevisiae. Aging Cell 8: 353-369, 2009.
20. Lu JM, Lin PH, Yao Q and Chen C: Chemical and molecular mechanisms of antioxidants: experimental approaches and model systems. J Cell Mol Med 14: 840-860, 2010.

21. Wang Y, Liang Y and Vanhoutte PM: SIRT1 and AMPK in regulating mammalian senescence: a critical review and a working model. FEBS Lett 585: 986-994, 2011.

22. Kitada M, Kume S, Takeda-Watanabe A, Tsuda S, Kanasaki K and Koya D: Calorie restriction in overweight males ameliorates obesity-related metabolic alterations and cellular adaptations through anti-aging effects, possibly including AMPK and SIRT1 activation. Biochim Biophys Acta 1830: 4820-4827, 2013.

23. Salminen A and Kaarniranta K: AMP-activated protein kinase (AMPK) controls the aging process via an integrated signaling network. Ageing Res Rev 11: 230-241, 2012.

24. Hardie DG, Ross FA and Hawley SA: AMPK: a nutrient and energy sensor that maintains energy homeostasis. Nat Rev Mol Cell Biol 13: 251-262, 2012.

25. Reznick RM, Zong H, Li J, et al: Aging-associated reductions in AMP-activated protein kinase activity and mitochondrial biogenesis. Cell Metab 5: 151-156, 2007.

26. Stenesen D, Suh JM, Seo J, et al: Adenosine nucleotide biosynthesis and AMPK regulate adult life span and mediate the longevity benefit of caloric restriction in flies. Cell Metab 17: 101-112, 2013.

27. Tanno M, Kuno A, Yano T, et al: Induction of manganese superoxide dismutase by nuclear translocation and activation of SIRT1 promotes cell survival in chronic heart failure. J Biol Chem 285: 8375-8382, 2010.

28. Tong C, Morrison A, Mattison S, et al: Impaired SIRT1 nucleocytoplasmic shuttling in the senescent heart during ischemic stress. FASEB J 27: 4332-4342, 2013.

29. Chainiaux F, Magalhaes JP, Eliaers F, Remacle J and Toussaint O: UVB-induced premature senescence of human diploid skin fibroblasts. Int J Biochem Cell Biol 34: 1331-1339, 2002.

30. Chung KW, Choi YJ, Park MH, et al: Molecular insights into SIRT1 protection against UVB-induced skin fibroblast senescence by suppression of oxidative stress and p53 acetylation. J Gerontol A Biol Sci Med Sci: Aug 27, 2014 (Epub ahead of print).

31. Yoo HG, Lee BH, Kim W, et al: Lithospermum erythrorhizon extract protects keratinocytes and fibroblasts against oxidative stress. J Med Food 17: 1189-1196, 2014.

32. Hwang E,Lee TH,Park SY,YiTH and Kim SY: Enzyme-modified Panax ginseng inhibits UVB-induced skin aging through the regulation of procollagen type I and MMP-1 expression. Food Funct 5: 265-274, 2014

33. Kampkötter A, Pielarski T, Rohrig R, et al: The Ginkgo biloba extract EGb761 reduces stress sensitivity, ROS accumulation and expression of catalase and glutathione S-transferase 4 in Caenorhabditis elegans. Pharmacol Res 55: 139-147, 2007.

34. Gospodaryov DV, Yurkevych IS, Jafari M, Lushchak VI and Lushchak OV: Lifespan extension and delay of age-related functional decline caused by Rhodiola rosea depends on dietary macronutrient balance. Longev Healthspan 2: 5, 2013.

35. Burkewitz K, Zhang Y and Mair WB: AMPK at the nexus of energetics and aging. Cell Metab 20: 10-25, 2014.

36. Apfeld J, O'Connor G, McDonagh T, DiStefano PS and Curtis R: The AMP-activated protein kinase AAK-2 links energy levels and insulin-like signals to lifespan in C. elegans. Genes Dev 18: 3004-3009, 2004.

37. Greer EL, Dowlatshahi D, Banko MR, et al: An AMPK-FOXO pathway mediates longevity induced by a novel method of dietary restriction in C. elegans. Curr Biol 17: 1646-1656, 2007.

38. Greer EL, Oskoui PR, Banko MR, et al: The energy sensor AMP-activated protein kinase directly regulates the mammalian FOXO3 transcription factor. J Biol Chem 282: 30107-30119, 2007.

39. Schlernitzauer A, Oiry C, Hamad R, et al: Chicoric acid is an antioxidant molecule that stimulates AMP kinase pathway in L6 myotubes and extends lifespan in Caenorhabditis elegans. PLoS One 8: e78788, 2013.

40. Onken B and Driscoll M: Metformin induces a dietary restriction-like state and the oxidative stress response to extend C. elegans healthspan via AMPK, LKB1, and SKN-1. PLoS One 5: e8758, 2010.

41. Yin Y, Li W, Son YO, et al: Quercitrin protects skin from UVB-induced oxidative damage. Toxicol Appl Pharmacol 269: 89-99, 2013.

42. Eid HM, Martineau LC, Saleem A, et al: Stimulation of AMP-activated protein kinase and enhancement of basal glucose uptake in muscle cells by quercetin and quercetin glycosides, active principles of the antidiabetic medicinal plant Vaccinium vitis-idaea. Mol Nutr Food Res 54: 991-1003, 2010. 
43. Liu JF, Ma Y, Wang Y, Du ZY, Shen JK and Peng HL: Reduction of lipid accumulation in HepG2 cells by luteolin is associated with activation of AMPK and mitigation of oxidative stress. Phytother Res 25: 588-596, 2011

44. Cohen HY, Miller C, Bitterman KJ, et al: Calorie restriction promotes mammalian cell survival by inducing the SIRT1 deacetylase. Science 305: 390-392, 2004.

45. Bordone L, Cohen D, Robinson A, et al: SIRT1 transgenic mice show phenotypes resembling calorie restriction. Aging Cell 6: 759-767, 2007.

46. Finkel T and Holbrook NJ: Oxidants, oxidative stress and the biology of ageing. Nature 408: 239-247, 2000.

47. Lam YY, Peterson CM and Ravussin E: Resveratrol vs. calorie restriction: data from rodents to humans. Exp Gerontol 48: 1018-1024, 2013.

48. Amić D, Davidović-Amić D, Beslo D, Rastija V, Lucić B and Trinajstić N: SAR and QSAR of the antioxidant activity of flavonoids. Curr Med Chem 14: 827-845, 2007.
49. Cheng SC, Li WH, Shi YC, et al: Antioxidant activity and delayed aging effects of hot water extract from Chamaecyparis obtusa var. formosana leaves. J Agric Food Chem 62: 4159-4165, 2014.

50. Heim KE, Tagliaferro AR and Bobilya DJ: Flavonoid antioxidants: chemistry, metabolism and structure-activity relationships. J Nutr Biochem 13: 572-584, 2002.

51. Comalada M, Camuesco D, Sierra S, et al: In vivo quercitrin anti-inflammatory effect involves release of quercetin, which inhibits inflammation through down-regulation of the NF-kappaB pathway. Eur J Immunol 35: 584-592, 2005.

52. Jiang S, Yang J, Qian D, et al: Rapid screening and identification of metabolites of quercitrin produced by the human intestinal bacteria using ultra performance liquid chromatography/quadrupole-time-of-flight mass spectrometry. Arch Pharm Res 37: 204-213, 2014. 